\section{RA-Behandlung mit Tocilizumab erhöht Risiko von Herz- und Gefäßkomplikationen}

\author{
Giles JT et al. Cardiovascular Safety of Tocili- \\ zumab versus Etanercept in Rheumatoid \\ Arthritis: A Randomized Controlled Trial. Arthritis \\ Rheumatol 2020; 72: 31-40
}

In Studien konnte beobachtet werden, dass die Therapie von rheumatoider Arthritis (RA) mit Tocilizumab die Blutfettwerte der behandelten Patienten erhöht. Die erhöhten Konzentrationen von Blutfetten stellen nun das RisikoNutzen-Verhältnis von Tocilizumab zur
Behandlung einer RA in Frage. Giles et al. evaluierten das Risiko kardiovaskulärer Ereignisse bei der Behandlung mit Tocilizumab im Vergleich zum TNF-Inhibitor Etanercept.

Die Ergebnisse dieser Studie zeigen, dass größere unerwünschte kardiovaskuläre Ereignisse, die durch die Behandlung mit Tocilizumab auftreten können, im Vergleich zur Behandlung mit Etanercept ein relatives Risiko von 1,43 nicht übersteigen. Die Wissenschaftler führten eine randomisierte Multicenterstudie, im Rahmen derer sie Patienten mit per American College of Rheumatology-Kriterien diagnostizierten RA-Patienten einschlossen, die eine Krankheitsdauer von $\geq 6$ Monaten aufwiesen und unzureichend auf konventionelle synthetische krankheitsverändernde Antirheumatika oder anti-Tumornekrosefaktor-Therapeutika ansprachen. Außerdem mussten die Patienten unter anderem seropositiv auf den Rheumafaktor sein oder Antikörper gegen zyklisches citrulliniertes Peptid aufweisen, $\geq 8$ geschwollene und empfindliche Gelenke haben, einen C-reaktiven ProteinSerumspiegel $>0,3 \mathrm{mg} / \mathrm{L}$ aufweisen und mindestens einen kardiovaskulären Risikofaktor haben. Die Forscher randomisierten die Patienten 1:1 in die Studiengruppen zur Behandlung mit Tocilizumab oder Etanercept. Die Patienten der Tocilizumab-Gruppe erhielten alle 4 Wochen $8 \mathrm{mg} / \mathrm{kg}$ intravenös verabreichtes Tocilizumab, die Patienten der Etanercept-Gruppe einmal wöchentlich $50 \mathrm{mg}$ subkutan verabreichtes Etanercept. Proben von peripherem Blut entnahmen die Experten jeweils einen und 3 Monate nach Studienbeginn, anschließend bis zum dritten Jahr der Studie alle 3 Monate und ab dem vierten Jahr alle 6 Monate. Die Forscher bestimmten die Werte des Gesamtcholesterins, der Blut-Triglyceride, des HDL- und des LDL-Cholesterins. Als primären Endpunkt definierten die Wissenschaftler die Zeit bis zum ersten Auftreten größerer unerwünschter kardiovaskulärer Ereignisse.

Insgesamt 1538 Patienten ordneten die Forscher dem Tocilizumab-Arm, 1,542 dem Etanercept-Arm zu. Über eine Nachbeobachtungszeit von 3,2 Jahren konnten $96 \%$ der eingeschlossenen Patienten die Studie mit einer vollständigen Evaluierung der kardiovaskulären Krankheitsvorfälle abschlie- 
Ben. Bereits 4 Wochen nach Studienbeginn wies die Tocilizumab-Gruppe im Mittel einen Anstieg um 11,1 \% des LDL-Cholesterinspiegels, einen Anstieg von 5,4\% des HDL-Cholesterinspiegels und einen Anstieg von 13,6\% der Triglycerid-Werte im Vergleich zur Etanercept-Gruppe auf. Diese Effekte hielten auch im Laufe der Zeit an. Bei insgesamt 161 Patienten traten größere unerwünschte kardiovaskuläre Ereignisse auf, davon passierten 78 in der EtanerceptGruppe und 83 in der Tocilizumab-Gruppe. Im Vergleich zwischen Tocilizumab und Etanercept betrug der Risikoquotient für den primären Endpunkt der unerwünschten kardiovaskulären Ereignisse, zu denen auch unbestimmte Todesursachen gezählt wurden, 1,05.

\section{FAZIT}

Die Resultate dieser Studie unterstreichen den Anstieg an Blutfettwerten bei einer RA-Therapie mit Tocilizumab. Unerwünschte kardiovaskuläre Ereignisse kamen im Vergleich zur Etanercept-Gruppe häufiger im Studienarm vor, der mit Tocilizumab behandelt wurde. Die Autoren schlossen ein Risiko von 1,43 oder höher für das Auftreten größerer unerwünschter kardiovaskulärer Ereignisse bei den mit Tocilizumab behandelten Patienten allerdings aus.

Dr. Maddalena Angela Di Lellis, Tübingen 